\title{
Thymoma with idiopathic thrombocytopenic purpura: Report of a case
}

\author{
Jianjun Qin, MD, ${ }^{a}$ and Lunxu Liu, MD, ${ }^{\mathrm{b}}$ Zhengzhou and Sichuan, People's Republic of China
}

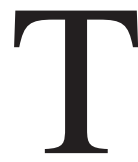

hymoma is often accompanied by myasthenia gravis pure red cell aplasia because the thymus is an immune organ. Idiopathic thrombocytopenic purpura (ITP) is also an autoimmune disease; however, only rarely is thymoma followed by ITP. We report the case of a patient with thymoma and chronic ITP who was helped by thymectomy.

\section{Clinical Summary}

A 27-year-old woman with repeated limb petechiae, epistaxis, and menorrhagia was admitted to the hospital. She was treated for 4 months in the department of internal medicine. At first, decreased serum platelet count $\left(13.0 \times 10^{9}\right.$ cells/L) was detected. Erythrocyte count was almost within normal limits. The result of bone marrow examination was consistent with ITP. Prednisone, given orally for 4 months, was effective. Because the patient coincidentally reported discomfort in the chest, thymoma was detected by radiography and computed tomography (Figure 1). Thymectomy was performed. Prednisone was continuously applied in the postoperative period, but the dose was gradually decreased. ITP was improved. Three months later, corticosteroid therapy was no longer used. Platelet counts were normal. During a 1-year follow-up period, ITP did not recur, and the patient appears to be cured.

\section{Discussion}

Primary immune thrombocytopenic purpura, as ITP is also known, is an organ-specific autoimmune disorder in which antibodycoated or immune complex-coated platelets are destroyed prematurely by the reticuloendothelial system, resulting in peripheral blood thrombocytopenia. The disease is heterogeneous with regard to its severity and clinical course and is unpredictable in its

\footnotetext{
From the Department of Thoracosurgery, The Affiliated Hospital, Zhengzhou University, Zhengzhou, ${ }^{\text {a }}$ and the Department of Thoracosurgery, West-China Hospital, Sichuan University, Sichuan, ${ }^{\text {b }}$ People's Republic of China.

Received for publication May 16, 2004; accepted for publication June 3, 2004.

Address for reprints: Qin Jianjun, MD, Department of Thoracosurgery, The Affiliated Hospital, Zhengzhou University, Zhengzhou 450052, People's Republic of China (E-mail: qinjianjun@yahoo.com.cn).

J Thorac Cardiovasc Surg 2005;129:453

$0022-5223 / \$ 30.00$

Copyright (C) 2005 by The American Association for Thoracic Surgery doi:10.1016/j.jtcvs.2004.06.021
}

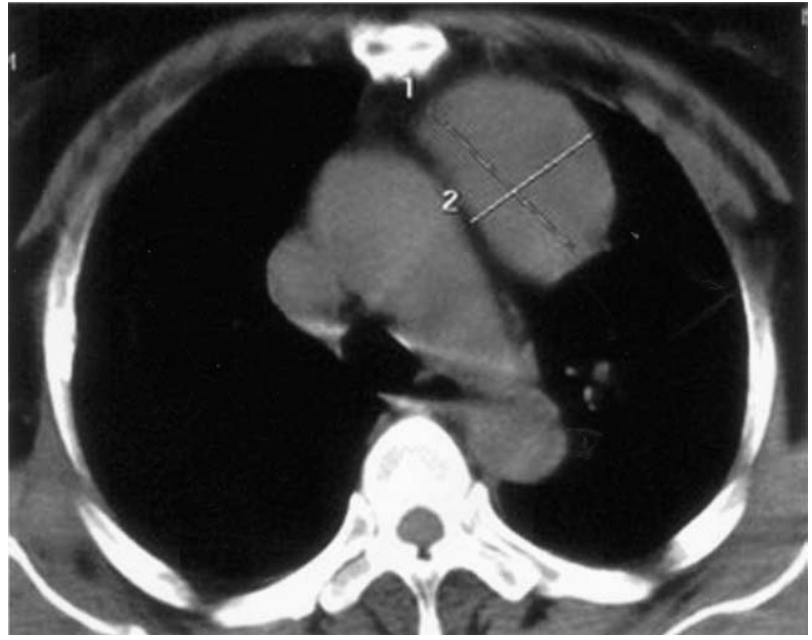

Figure 1. Computed tomographic scan demonstrates thymoma. 1, length; 2, breadth.

response to therapy. ${ }^{1}$ It is generally thought that antibody-coated platelets are produced in the spleen. Treatment of patients with ITP refractory to corticosteroids and splenectomy requires careful evaluation of disease severity, patient characteristics related to risk of bleeding, and adverse effects associated with treatment. ITP in adults does not generally remit spontaneously, and most patients require treatment to prevent bleeding at one time or another. ITP combined with thymoma is even rarer. Previous reports noted no certain relation between thymoma and ITP, and ITP was not improved after thymectomy. ${ }^{2}$ In our case, however, a thorough cure was effected by thymectomy. Apparently her myasthenia gravis was cured by thymectomy, which also differs from previous reports. ${ }^{2,3}$ We therefore speculate that the thymus was engaged in the pathogenesis of ITP in this case, although we do not know this for certain.

\section{References}

1. Stasi R, Provan D. Management of immune thrombocytopenic purpura in adults. Mayo Clin Proc. 2004;79:504-22.

2. Matsuge S, Hosokawa Y, Murakami Y, Satoh K. Thymoma with idiopathic thrombocytopenic purpura preceded by mediastinal hemorrhage; report of a case [Japanese]. Kyobu Geka. 2002;55:899-902.

3. Kobayashi H, Kitano K, Ishida F, Saito H, Miyabayashi H, Yonezawa $\mathrm{T}$, et al. Aplastic anemia and idiopathic thrombocytopenic purpura with antibody to platelet glycoprotein IIb/IIIa following resection of malignant thymoma. Acta Haematol. 1993;90:42-5. 